\title{
FIXED POINT THEOREMS FOR SEMI-GROUPS OF SELF MAPS OF SEMI-METRIC SPACES
}

\section{G. JUNGCK}

Department of Mathematics

Bradley University

Peoria, Il, 61625

(Received May 2, 1996 and in revised form January 2, 1997)

\begin{abstract}
We use selected semi-groups of self maps of a semi-metric space to obtain fixed point theorems for single maps and for families of maps - theorems which generalize results by Browder, Jachymski, Rhoades and Walters, and others. A basic tool in our approach is the concept of commuting maps.
\end{abstract}

KEY WORDS AND PHRASES: Semi-metric, semi-group, commuting maps, fixed points. 1991 AMS SUBJECT CLASSIFICATION CODES: Primary 54E25, 54H25.

1. INTRODUCTION. By a semi-group of maps we shall mean a family $\mathrm{H}$ of self maps of a set $\mathrm{X}$ which is closed with respect to composition of maps. Thus, if $f, g \in H$, then $f \circ g \in H$. Since composition of maps is associative, $\mathrm{H}$ is indeed a semi-group with respect to composition. We shall write $f g$ for $f \circ g$ and $f x$ for $f(x)$ when convenient and confusion is not likely.

We shall utilize the following semi-groups of maps in subsequent sections.

1.1. Let $g \cdot X \rightarrow X$, and $H=O_{g}=\left\{g^{n}: n \in N \cup\{0\}\right\}$, where $N$ is the set of positive integers, $g^{0}=i_{d}-$ the identity map, $g^{l}=g$ and $g^{n+1}=g \circ g^{n}$.

1.2. Let $g: X \rightarrow X$, and $H=C_{g}=\{f: X \rightarrow X \mid f g=g f\}$. $C_{g}$ is a semi-group. For if $f, h \in C_{g}$, then $(f h) g=f(h g)=f(g h)=(f g) h=(g f) h=g(f h)$, and thus, $f h \in C_{g}$.

1.3. $\mathrm{H}=\left\{\mathrm{i}_{\mathrm{d}}\right\}$, and

1.4 If $f, g: X \rightarrow X$ and $f g=g f$, we can let $H=\left\{f^{n} g^{m}: n, m \in N \cup\{0\}\right\}$.

If $H$ is a semi-group of self maps of a set $X$ and $a \in X$, then $H(a)=\{h(a): h \in H\}$.

Consequently, if $g: X \rightarrow X$ and $H=O_{g}, O_{g}(a)=\left\{g^{n}(a): n \in N \cup\{0\}\right\}$, and is called the orbit of $g$ at $a$.

Just as we use semi-groups of maps to generalize the concept of orbits, we shall use semi-metric spaces to generalize results pertaining to metric spaces. We need the following definitions.

DEFINITION 1.1 A symmetric on a set $X$ is a function $d: X x X \rightarrow[0, \infty)$ such that $d(x, y)$ $=0$ iff $x=y$ and $d(x, y)=d(y, x)$ for all $x, y \in X$.

Given a symmetric $d$ on a set $X$, we generate an induced topology $t(d)$ for $X$ as follows. For $x \in X$ and $\epsilon>0$, we let $S_{\epsilon}(x)=\{y \in X: d(x, y)<\epsilon\}$. Then $t(d)$ consists of all subsets $U$ of $X$ such that for each $p \in U, S_{\epsilon}(p) \subseteq U$ for some $\epsilon>0$. Just as in the case of a metric, $t(d)$ is a topology on $X$. However, if $d$ is a symmetric, the sets $S_{\epsilon}(x)$ need not be neighborhoods of $x$. A semi-metric is a symmetric $d$ such that all sets $S_{\epsilon}(x)$ are neighborhoods of $x$; i.e., $\exists U \in t(d)$ such that $x \in U \subseteq S_{\epsilon}(x)$. It 
is easy to verify that if $d$ is a semi-metric, then a sequence $\left\{x_{n}\right\}$ in $X$ converges to $x \in X$ in the topology $t(d)$ iff $d\left(x_{n}, x\right) \rightarrow 0$. This is the property we desire. Hence, the following terminology.

DEFINITION 1.2 A semi-metric space is a topological space, denoted by $(X ; d)$, with topology $t(d)$ induced on the set $X$ by a semi-metric $d$.

For further discussion of symmetrics and semi-metrics refer to [1] or [2]. In this context, we note that a semi-metric need not be Hausdorff (or $\mathrm{T}_{2}$ ) Example 2.2 in [2] gives an instance of such a semimetric. Since we desire uniqueness of limits, we shall in most instances require that a semi-metric space (X; d) be Hausdorff. Note also that - as in metric spaces - we shall say a semi-metric space $(X ; d)$ is complete iff every Cauchy sequence in $\mathrm{X}$ converges to a point in $\mathrm{X}$. If $\mathrm{g}: \mathrm{X} \rightarrow \mathrm{X}$, then $(\mathrm{X} ; \mathrm{d})$ is $\mathrm{g}$ orbitally complete iff every Cauchy sequence in $\mathrm{O}_{\mathrm{g}}(\mathrm{x})$ converges to a point in $\mathrm{X}$ for all $\mathrm{x} \in \mathrm{X}$. A function $F . X \rightarrow[0, \infty)$ is lower semicontinuous iff $F(x) \leq \lim _{n \rightarrow \infty} \inf F\left(x_{n}\right)$ when $\left\{x_{n}\right\}$ is a sequence in $X$ converging to $x$.

To produce fixed points we use a contractive function $\mathrm{P}:[0, \infty) \rightarrow[0, \infty)$ which is nondecreasing and which satisfies: $\lim _{n \rightarrow \infty} P^{n}(t)=0$ for each $t \in[0, \infty)$. Throughout this paper, $P$ will denote such a map, and $\mathcal{P}$ will denote the family of all such maps $P$

2. FLXED POINT THEOREMS. The major results in this paper evolve from the following lemma.

LEMMA 2.1. Let $\mathrm{X}$ be a set, $\mathrm{g}: \mathrm{X} \rightarrow \mathrm{X}$, and let $\mathrm{d}: \mathrm{XXX} \rightarrow[0, \infty)$. Let $\mathrm{H}$ be a semi-group of maps $h X \rightarrow X$ such that $H \subset C_{g}$ Suppose that for each pair $X, y \in X$ there is a choice of $r=r(x, y)$, $\mathrm{s}=\mathrm{s}(\mathrm{x}, \mathrm{y}) \in \mathrm{H}$ and $\mathrm{u}, \mathrm{v} \in\{\mathrm{x}, \mathrm{y}\}$ for which

(i) $\mathrm{d}(\mathrm{gx}, \mathrm{gy}) \leq \mathrm{P}(\mathrm{d}(\mathrm{ru}, \mathrm{sv}))$.

Then, if $n \in N$, for each pair $x, y \in X \exists r_{n}, s_{n} \in H$ and $u_{n}, v_{n} \in\{x, y\}$ such that

(ii) $d\left(g^{n} x, g^{n} y\right) \leq P^{n}\left(d\left(r_{n} u_{n}, s_{n} v_{n}\right)\right)$.

PROOF. (ii) holds for $n=1$ by (i), so suppose $n \in N$ for which (ii) is true. Then, if $x, y \in X$,

$$
\begin{aligned}
& d\left(g^{n+1} x, g^{n+1} y\right)=d\left(g\left(g^{n} x\right), g\left(g^{n} y\right)\right) \leq P(d(r u, s v)), \\
& \text { where } r, s \in H \text { and } u, v \in\left\{g^{n} x, g^{n} y\right\}, \text { by (i). }
\end{aligned}
$$

Specifically, $u=g^{n} u_{0}$, where $u_{0} \in\{x, y\}$ and $v=g^{n} v_{0}$ with $v_{o} \in\{x, y\}$. Thus

$$
\begin{gathered}
d(r u, s v)=d\left(r\left(g^{n} u_{o}\right), s\left(g^{n} v_{o}\right)\right) \text { where } u_{o}, v_{o} \in\{x, y\} \text {. And since } r, s \in H \subset C_{g} \text {, } \\
d(r u, s v)=d\left(g^{n}\left(r u_{o}\right), g^{n}\left(s v_{o}\right)\right) \leq P^{n}\left(d\left(r_{n} u_{n}, s_{n} v_{n}\right)\right) \text {, by (ii), }
\end{gathered}
$$

where $r_{n}, s_{n} \in H$ and $u_{n}, v_{n} \in\left\{r_{0}, s v_{0}\right\}$.

Then $r_{n} u_{n} \in\left\{\left(r_{n} r\right) u_{0},\left(r_{n} s\right) v_{0}\right\}$, where $r_{n} r, r_{n} s \in H$ (a semi-group). So, $r_{n} u_{n}=r_{n+1} u_{n+1}$, where $r_{n+1} \in H$ (i.e., $\left.r_{n+1} \in\left\{r_{n} r, r_{n} s\right\}\right)$ and $u_{n+1} \in\left\{u_{0}, v_{0}\right\} \subset\{x, y\}$. Similary, $s_{n} v_{n}=s_{n+1} v_{n+1}$, where $s_{n+1} \in H$ and $v_{n+1} \in\{x, y\}$. Thus (2.2) implies

$$
d(r u, s v) \leq P^{n}\left(d\left(r_{n+1} u_{n+1}, s_{n+1} v_{n+1}\right)\right) \text {, with } r_{n+1}, s_{n+1} \in H \text { and } u_{n+1}, v_{n+1} \in\{x, y\} \text {. }
$$

But $P$ is nondecreasing; therefore, (2.1) and (2.3) imply

$$
d\left(g^{n+1} x, g^{n+1} y\right) \leq P\left(P^{n}\left(d\left(r_{n+1} u_{n+1}, s_{n+1} v_{n+1}\right)\right)=P^{n+1}\left(d\left(r_{n+1} u_{n+1}, s_{n+1} v_{n+1}\right),\right.\right.
$$

with $r_{n+1}, s_{n+1} \in H$ and $u_{n+1}, v_{n+1} \in\{x, y\}$. Thus, (ii) is true for all $n$, by induction.

THEOREM 2.1 Let $(X ; d)$ be a $T_{2}$ semi-metric space. Let $g: X \rightarrow X$ and let $(X ; d)$ be $g$ orbitally complete. Suppose $H$ is a semi-group of self maps of $X$ such that $H \subset C_{g}$, and there is an $a \in X$ for which $H(a)$ is bounded and $g(H(a)) \subset H(a)$. If for each $x, y \in X \quad \exists a$ choice of $r, s \in H$ and $u, v \in\{x, y\}$ such that

$$
\left(^{*}\right) d(g x, g y) \leq P(d(r u, s v)),
$$


then $g^{n}(a) \rightarrow c$ for some $c \in X$. If $g$ is continuous at $c$, then $g(c)=c$. If $d$ is lower semicontinuous, then $c$ is a fixed point for all $h(\in H)$ continuous at $c$. Moreover, if $g$ and each $h \in H$ are continuous at $c$, then $\mathrm{c}$ is the unique common fixed point of $\mathrm{g}$ and the family $\mathrm{H}$.

PROOF. We first prove that $\left\{g^{n}(a)\right\}$ is Cauchy. By Lemma 2.1., for each pair $n, k \in N$, there is a choice of $r_{n}, S_{n} \in H$ and $u_{n}, v_{n} \in\left\{a, g^{k} a\right\}$ such that

$$
d\left(g^{n}(a), g^{n+k}(a)\right)=d\left(g^{n}(a), g^{n}\left(g^{k}(a)\right) \leq P^{n}\left(d\left(r_{n} u_{n}, s_{n} v_{n}\right)\right) .\right.
$$

Now $r_{n} \in H$ implies $r_{n} a \in H(a)$ and $r_{n} g^{k}(a)=g^{k}\left(r_{n} a\right) \in H(a)$, since $g(H(a)) \subseteq H(a)$ implies that $g^{k}(H(a)) \subseteq H(a)$. Thus $r_{n} u_{n} \in H(a)$. Similarly, $s_{n} v_{n} \in H(a)$. But $H(a)$ is bounded, so $\exists M \geq 0$ such that $d(x, y) \leq M$ for $x, y \in H(a)$. Thus, $d\left(r_{n} u_{n}, s_{n} v_{n}\right) \leq M$ for $n \in N$. Then (2.4) implies

$$
d\left(g^{n}(a), g^{n+k}(a)\right) \leq P^{n}(M) \text {, for } n, k \in N
$$

since $P$ is nondecreasing. But $P^{n}(M) \rightarrow 0$ as $n \rightarrow \infty$. So given $\epsilon>0, \exists n_{0} \in N$ such that for any $m>n \geq n_{0}, \quad(2,5)$ implies $d\left(g^{n}(a), g^{m}(a)\right) \leq P^{n}(M)<\epsilon$, with $m=n+k$. Consequently, $\left\{g^{n}(a)\right\}$ is Cauchy.

Since $(X ; d)$ is g-orbitally complete, $g^{n}(a), g^{n+1}(a) \rightarrow c$ for some $c \in X$. If $g$ is continuous, $g\left(g^{n}(a)\right)=g^{n+1}(a) \rightarrow g(c)$; thus, $c=g(c)$ since $X$ is Hausdorff.

Now suppose that $d$ is lower semicontinuous and that $h(\in H)$ is continuous at $c$. Then, since $\mathrm{H} \subseteq \mathrm{C}_{\mathrm{B}}$ and $\mathrm{g}^{\mathrm{n}}(\mathrm{a}) \rightarrow \mathrm{c}$,

$$
\mathrm{g}^{\mathrm{n}}(\mathrm{h}(\mathrm{a}))=\mathrm{h}\left(\mathrm{g}^{\mathrm{n}}(\mathrm{a})\right) \rightarrow \mathrm{h}(\mathrm{c}) .
$$

But $\left(^{*}\right)$ and Lemma 2.1 tell us that $\exists r_{n}, s_{n} \in H$ and $u_{n}, v_{n} \in\{a, h(a)\}$ such that

$$
d\left(g^{n}(a), g^{n}(h(a))\right) \leq P^{n}\left(d\left(r_{n} u_{n}, s_{n} v_{n}\right)\right) .
$$

Then $r_{n} u_{n}=r_{n} a \in H(a)$ or $r_{n} u_{n}=r_{n} h(a) \in H(a) \quad\left(r_{n} h \in H\right.$, since $H$ is a semi-group.). Thus, in either event, $r_{n} u_{n} \in H(a)$. In like manner, we conclude that $s_{n} v_{n} \in H(a)$. Then, as above, $d\left(r_{n} u_{n}, s_{n} v_{n}\right) \leq M$ for $n \in N$, which implies by (2.7)

$$
\mathrm{d}\left(\mathrm{g}^{\mathrm{n}}(\mathrm{a}), \mathrm{g}^{\mathrm{n}}(\mathrm{h}(\mathrm{a}))\right) \leq \mathrm{P}^{\mathrm{n}}(\mathrm{M}) \rightarrow 0 .
$$

But since $g^{n}(a) \rightarrow c,(2.6)$ implies that $\left(g^{n}(a), g^{n}(h(a))\right) \rightarrow(c, h(c))$ in $X x X$. Since $d$ is lower semicontinuous, $d(c, h(c)) \leq \lim _{n \rightarrow \infty} d\left(g^{n}(a), g^{n}(h(a))\right)=0$ by $(2.8)$, so $h(c)=c$.

To complete the proof we have yet to show that if $\mathrm{c}$ is a common fixed point for $\mathrm{g}$ and every $h \in H$, then $c$ is the only such point. So suppose that $z \in X$ and that $z=g(z)=h(z)$ for all $h \in H$. Then by $\left({ }^{*}\right)$ and Lemma 3.1 , we can write:

$$
d(c, z)=d\left(g^{n}(c), g^{n}(z)\right) \leq P^{n}\left(d\left(r_{n} u_{n}, s_{n} v_{n}\right)\right)
$$

where $r_{n}, s_{n} \in H$ and $u_{n}, v_{n} \in\{c, z\}$. But then $r_{n} u_{n} \in\{c, z\}$. Similarly, $s_{n} v_{n} \in\{c, z\}$. Therefore, $d\left(r_{n} u_{n}, s_{n} v_{n}\right)=0$ or $d(c, z)$. Thus (2.9) says that $d(c, z) \leq P^{n}(d(c, z))$. Since $P^{n}(d(c, z)) \rightarrow 0, c=z$; i.e., $\mathrm{c}$ is unique.

The following example shows that the family $\mathrm{H}$ in Theorem 2.1 can have fixed points other than the unique common fixed point of $\mathrm{g}$ and $\mathrm{H}$.

EXAMPLE 2.1. Let $X=\{0,1\}, g(x)=0$ for $x \in X \quad h(x)=x$ for $x \in X$. Let $d(x, y)=|x-y|$ and $H=\left\{h^{n}: n \in N\right\}$. Since $h^{n}(x)=x$ for $n \in N, H=\left\{i_{d}\right\}$. Since $d(g x, g y)=0$ for all $x, y \in X$, it is immediate that $\mathrm{g}$ and $\mathrm{H}$ satisfy the hypothesis of Theorem 3.1 with $\mathrm{a}=0$, and $\mathrm{l}$ is a fixed point of $\mathrm{H}$ but not of $g$.

The first corollary provides conditions necessary and sufficient to ensure that a family $\mathrm{H}$ of continuous self maps of a semi-metric space has a fixed point. 
COROLLARY 2.1. Let $(X ; d)$ be a complete Hausdorff semi-metric space with $d$ lower semicontinuous. A semi-group $H$ of continuous self maps of $X$ has a common fixed point iff $H(a)$ is bounded for some $\mathrm{a} \in \mathrm{X}$, and $\exists \mathrm{P} \in \mathcal{P}$ and a continuous self map $\mathrm{g}$ of $\mathrm{X}$ which satisfies the following.

1. $\mathrm{H} \subseteq \mathrm{C}_{\mathrm{B}}$ and $\mathrm{g}(\mathrm{H}(\mathrm{a})) \subseteq \mathrm{H}(\mathrm{a})$

2. For any $x, y \in X, \exists$ a choice of $r, s \in H$ and $u, v \in\{x, y\}$ such that $\mathrm{d}(\mathrm{gx}, \mathrm{gy}) \leq \mathrm{P}(\mathrm{d}(\mathrm{ru}, \mathrm{sv}))$.

PROOF. That the conditions are sufficient follows immediately from Theorem 2.1.. To prove necessity, suppose $a \in X$ and that $h(a)=a$ for $h \in H$. Then $H(a)=\{a\}$ and is thus bounded. Let $g(x)=$ a for $x \in X$. It is immediate that $g h=h g$ for all $h \in H$, so $H \subseteq C_{g}$. Moreover, $g(h(a))=a$ for all $h \in H$, so that $g(H(a)) \subseteq H(a)$ and statement 1 . of the Corollary holds. Statement 2. follows upon noting that $d(g x, g y)=d(a, a)=0$ for all $x, y \in X$. (We can let $P(t)=t / 2$, e.g.)

NOTE 2.1. The next result and proof suggest that the function $g$ of Theorem 2.1 may have an infinitude or unbounded set of fixed points, although $\mathrm{H}$ may have just one. Example 3.1 in the next section confirms this.

COROLLARY 2.2. Let $(X ; d)$ be a complete Hausdorff semi-metric space with $d$ lower semicontinuous. A semi-group $H$ of continuous self maps of $X$ has a common fixed point provided $\exists$ $a \in X$ such that $H(a)$ is bounded, and for any $x, y \in X \exists r, s \in H$ and $u, v \in\{x, y\}$ such that

$$
d(x, y) \leq P(d(r u, s v)) \text {. }
$$

PROOF. Let $g=i_{d}$, the identity map.

COROLLARY 2.3. Let $g$ be a self map of a metric space $(X, d)$ which is g-orbitally complete. If $\exists a \in X$ such that $O_{g}(a)$ is bounded and $k \in N$ such that for each pair $x, y \in X$ there is a choice of $n=n(x, y), m=m(x, y) \in N$ and $u, v \in\{x, y\}$, for which

$\left({ }^{* \prime}\right) \quad d\left(g^{k} x, g^{k} y\right) \leq P\left(d\left(g^{n} u, g^{m} v\right)\right)$

then $g^{n}(a) \rightarrow c$ for some $c \in X$. Moreover, if $x_{0} \in X$ and $O_{g}\left(x_{0}\right)$ is bounded, then $g^{n}\left(x_{0}\right) \rightarrow c$. If $g$ is continuous at $\mathrm{c}, \mathrm{c}$ is the unique fixed point of $\mathrm{g}$.

PROOF. Let $\mathrm{H}=\mathrm{O}_{8}$. Note that $\mathrm{H} \subseteq \mathrm{C}_{\mathrm{g}^{k}}, \mathrm{~g}(\mathrm{H}(\mathrm{a}))=\mathrm{g}\left(\mathrm{O}_{8}(\mathrm{a})\right) \subseteq \mathrm{O}_{8}(\mathrm{a})$, and that $\mathrm{g}^{\mathrm{k}}(\mathrm{H}(\mathrm{a}))$ $\subseteq H(a)$. Since $d$ is a metric, $d$ is actually uniformly continuous [3]. Thus, $g^{k n}(a) \rightarrow c$ as $n \rightarrow \infty$ for some $c \in X$, by Theorem 2.1 applied to $g^{k}$. If $g$ is continuous at $c$, each $g^{n} \in H\left(=O_{g}\right)$ is continuous at c. So, as an element of $\mathrm{H}, \mathrm{g}(\mathrm{c})=\mathrm{c}$ by Theorem 2.1 ..

We have yet to prove that $g^{n}(a) \rightarrow c$ and that $g^{n}\left(x_{0}\right) \rightarrow c$ for $x_{0}$ with $O_{g}\left(x_{0}\right)$ bounded. To see that $\mathrm{g}^{\mathrm{n}}(\mathrm{a}) \rightarrow \mathrm{c}$, let $\epsilon>0$. Since $\left(\mathrm{g}^{\mathrm{k}}\right)^{\mathrm{m}}(\mathrm{a}) \rightarrow \mathrm{c}$ as $\mathrm{m} \rightarrow \infty, \exists \mathrm{m}_{1} \in \mathrm{N}$ such that

$$
\mathrm{d}\left(\mathrm{g}^{\mathrm{km}}(\mathrm{a}), \mathrm{c}\right)<\epsilon / 2 \text {, for } \mathrm{m}>\mathrm{m}_{1}
$$

By Lemma 2.1 and $\left(^{* \prime}\right)$ of the hypothesis, if $m \in N$, for each pair $x, y \in X$ there exist $r_{m}, s_{m} \in H$ and $u_{m}, v_{m} \in\{x, y\}$ such that

$$
d\left(\left(g^{k}\right)^{m}(x),\left(g^{k}\right)^{m}(y)\right) \leq P^{m}\left(d\left(r_{m} u_{m}, s_{m} v_{m}\right)\right)
$$

Since $O_{B}(a)$ is bounded, $\exists M \geq 0$ such that

$$
d\left(r_{m} u_{m}, s_{m} v_{m}\right) \leq M \text { if } r_{m} u_{m} \text { and } s_{m} v_{m} \text { are in } O_{g}(a) \text {. }
$$

Now $\mathrm{P}^{\mathrm{m}}(\mathrm{M}) \rightarrow 0$ as $\mathrm{m} \rightarrow \infty$, so we can choose $m_{0} \in \mathrm{N}$ such that

$$
m_{0}>m_{1} \text { and } P^{m_{0}}(M)<\epsilon / 2 \text {. }
$$

Let $n>k m_{0}$. Then $n=k m_{0}+t_{n}$ for some $t_{n} \in N$, and (2.11) implies

$$
d\left(g^{n}(a), g^{k m_{0}}(a)\right)=d\left(g^{k m_{0}}\left(g^{h_{n}}(a)\right), g^{k m_{0}}(a)\right) \leq P^{m_{0}}\left(d\left(r_{m_{0}} u_{m_{0}}, s_{m_{0}} v_{m_{0}}\right)\right)
$$


where $r_{m_{0}} \in H=O_{g}, u_{m_{0}} \in\left\{a, g^{h}(a)\right\}$, so that $r_{m_{0}} u_{m_{0}} \in O_{g}(a)$. Similarly, $s_{m_{0}} v_{m_{0}} \in O_{g}(a)$.

Therefore, (2.12) implies that

$$
\begin{gathered}
d\left(r_{m_{0}} u_{m_{0}}, s_{m_{0}} v_{m_{0}}\right) \leq M \text {, and since } P \text { is nondecreasing, we have } \\
P^{m_{0}}\left(d\left(r_{m_{0}} u_{m_{0}}, s_{m_{0}} v_{m_{0}}\right)\right) \leq P^{m_{0}}(M)<\epsilon / 2 \text {, by (2.13). Thus (2.14) implies } \\
d\left(g^{n}(a), g^{k m_{0}}(a)\right)<\epsilon / 2 .
\end{gathered}
$$

But then (2.10) with $m=m_{0}$ and the triangle inequality imply that $d\left(g^{n}(a), c\right)<\epsilon$, since $m_{0}>m_{1}$. We therefore conclude that $\mathrm{g}^{\mathrm{n}}(\mathrm{a}) \rightarrow \mathrm{c}$

If $x_{0} \in X$ such that $O_{g}\left(x_{0}\right)$ is bounded, the above argument shows us that $\exists p \in X$ such that $g^{n}\left(x_{0}\right) \rightarrow p$. To see that $p=c$, first observe that $S=O_{g}\left(x_{0}\right) \cup O_{g}(a)$ is also bounded since $d$ is a metric; i.e., $\exists M_{0} \geq 0$ such that $d(x, y) \leq M_{0}$ if $x, y \in S$. We can therefore apply (*') and Lemma 2.1 as before to conclude that

$d(c, p)=\lim _{m \rightarrow \infty} d\left(g^{k m}(a), g^{k m}\left(x_{0}\right)\right) \leq \lim _{m} P^{m}\left(M_{0}\right)=0$

The following example shows that the hypothesis in Corollary 2.3 that the orbit $\mathrm{O}_{\mathrm{g}}(\mathrm{a})$ be bounded for at least one $a \in X$ is indeed necessary.

EXAMPLE 2.2. Let $X=[1, \infty), P(t)=t / 2$ for $t \in[0, \infty), d(x, y)=|x-y|$ and $g(x)=3 x$ for $x, y \in X$. Then $P^{n}(t)=t / 2^{n} \rightarrow 0$ as $n \rightarrow \infty, g: X \rightarrow X$ and $d(g x, g y)=|g x-g y|=3|x-y|$ $\leq 9 / 2|x-y|=1 / 2|9 x-9 y|=1 / 2\left|g^{2} x-g^{2} y\right|=P\left(d\left(g^{2} x, g^{2} y\right)\right)$. But since $g^{n}(x)=3^{n} x \rightarrow \infty$ for each $x \in X, O_{g}(x)$ is bounded for no $x \in X$ and $g$ has no fixed point.

In [4] Rhoades and Watson introduced the concept of a "generalized contraction".

DEFINITION 2.1 Let $(X, d)$ be a metric space. A function $\mathrm{f}: \mathrm{X} \rightarrow \mathrm{X}$ is a generalized contraction (with respect to $Q$ ) if $\exists p, q \in N$ such that for all $x, y \in X$

$$
d\left(f^{p} x, f^{q} y\right) \leq Q(M(x, y))
$$

where

$$
M(x, y)=\max \left\{d\left(f^{\prime} x, f^{j} y\right), d\left(f^{i} x, f^{i^{\prime}} x\right), d\left(f^{j} y, f^{j^{\prime}} y\right): 0 \leq i, i^{\prime} \leq p, 0 \leq j, j^{\prime} \leq q\right\} .
$$

( $Q$ is a nondecreasing function $Q:[0, \infty) \rightarrow[0, \infty)$ such that $Q(s)<s$ for $s>0$.)

NOTE 2.2. Jachymski [5] studied the relation (i) and observed that it satisfies

(ii) $\quad d\left(f^{\top} x, f^{\top} y\right) \leq Q\left(\max \left\{d\left(f^{\top} u, f^{j} v\right): 0 \leq i, j \leq r\right.\right.$ and $\left.\left.u, v \in\{x, y\}\right\}\right)$ where $r=\max \{p, q\}$, since $Q$ is nondecreasing. But (ii), and hence (i), satisfy the relation $\left({ }^{* \prime}\right)$ in Corollary 2.3. In fact, the following theorem by Jachymski-except the last sentence therein - is a consequence of Corollary 2.3. This last sentence refers to (9) which is essentially (i) above with the restriction that either $\mathrm{i}, \mathrm{i}^{\prime} \in\{0, \mathrm{p}\}$ or $\mathrm{j}, \mathrm{j}^{\prime} \in\{0, \mathrm{q}\}$.

THEOREM 4. ([5]) Let $f$ be a generalized contraction and let $(X, d)$ be f-orbitally complete. If $\lim _{n \rightarrow \infty} Q^{n}(s)=0$ for $s \in[0, \infty)$ and there exists a point $x_{0} \in X$ with a bounded orbit, then the sequence $\left\{f^{n} x_{0}\right\}$ converges to some $z \in X$. Moreover, for any $x \in X$ with a bounded orbit, $f^{n} x \rightarrow z$. Furthermore, if $f$ satisfies $(9) z$ is the unique fixed point of $f$.

The following example shows that if we use the more general contractive property $\left({ }^{* \prime}\right)$ of Corollary 2.3 , continuity at $c$ or restrictions of the ilk found in (9) of Theorem 4 are needed to ensure that the point c (or $z)$ is a fixed point.

EXAMPLE 2.3. Let $X=[0,1]$ and let $d(x, y)=|x-y|$. Define $g: X \rightarrow X$ by $g(x)=\frac{1}{2}(x+1)$ for $x \in[0,1)$ and $g(1)=\frac{1}{2}$. Then it is easy to see that $g^{n}(x)=(x-1) 2^{-n}+1(x \neq 1)$, and $g^{n}(1)=$ $1-2^{-n}$ for $n \in N$. Thus, $g^{n}(a) \rightarrow 1$ for any $a \in X$. Since $X$ is bounded, $O_{B}(a)$ is bounded for each $a \in X$. Thus, to see that the hypothesis of Corollary 2.3 is satisfied, we need only to verify that $\left({ }^{* \prime}\right)$ holds. A check shows that 


$$
d\left(g^{2} x, g^{2} y\right)=\frac{1}{2} d(g x, g y) \text { for all } x, y \in X .
$$

Thus, $\left({ }^{* \prime}\right)$ holds trivially with $k=2, n=m=1$, and $u=x, v=y$ for all $x, y$ in $[0,1]$. However, $g^{n}(a) \rightarrow 1$ for any a, but $g$ is not continuous at 1 and 1 is not a fixed point of $g$. Note that property (9) of Jachymski's Theorem 4. does not hold since in this instance $p=q=2$, and $g^{2}, g^{\circ}$ do not appear in the right member of (2.15).

3. THE BOUNDED CASE. The following is an example of a function $\mathrm{g}$ and a family $\mathrm{H}$ which satisfy the hypothesis of Theorem 2.1 and for which the set $F_{B}$ - the set of fixed points of $g$ - is not bounded We then consider the significance of this phenomenon.

EXAMPLE 3.1. Let $X=[0, \infty)$ and $d(x, y)=|x-y|$ for $x, y \in X$. Let $g(x)=x$ for $x \in X$; i.e., $g$ is the identity map. So $F_{g}=[0, \infty)$, and is unbounded. Let $P(t)=t / 2$ for $t \in[0, \infty)$ and define $h_{n}(x)=n x$ for $x \in X$ and $n \in N$. If $H=\left\{h_{n}: n \in N\right\}$, and $h_{n}, h_{m} \in H$, then $h_{n} h_{m}(x)=h_{n}\left(h_{m}(x)\right)=$ $h_{n}(m x)=(n m) x=h_{n m}(x)$. Thus $h_{n} h_{m}=h_{n m} \in H$, so that $H$ is indeed a semi-group. Since $g$ is the identity, the conditions $\mathrm{H} \subseteq \mathrm{C}_{\mathrm{B}}$ and $\mathrm{g}(\mathrm{H}(\mathrm{a})) \subseteq \mathrm{H}(\mathrm{a})$ ( for any $\mathrm{a} \in \mathrm{X}$ ) are satisfied trivially. Moreover, $d(g x, g y)=|x-y| \leq \frac{3}{2}|x-y|=\frac{1}{2}|3 x-3 y|=P\left(d\left(h_{3} x, h_{3} y\right)\right)$, so that $\left(^{*}\right)$ and hence the hypothesis of Theorem 2.1 is satisfied. $a=0$ is the unique fixed point for $\mathrm{g}$ and $\mathrm{H}$, but $\mathrm{g}$ has an infinitude of other , fixed points.

In the remainder of the paper, if $(X ; d)$ is a $T_{2}$ semi-metric space and $\mathrm{g}: \mathrm{X} \rightarrow \mathrm{X}$, we shall say that $g$ has property $P$ relative to a semi-group $H$ of self maps of $X$ iff for each pair $\mathrm{x}, \mathrm{y} \in \mathrm{X} \exists \mathrm{r}, \mathrm{s} \in \mathrm{H}$ and $\mathrm{u}, \mathrm{v} \in\{\mathrm{x}, \mathrm{y}\}$ such that

$\left(^{*}\right) \quad d(g x, g y) \leq P(d(r u, s v))$.

NOTE 3.1. If a function $\mathrm{g}: \mathrm{X} \rightarrow \mathrm{X}$ has property $\mathrm{P}$ relative to a semi-group $\mathrm{H}$ of self maps of $\mathrm{X}$ for which $H \subseteq C_{g}$, Lemma 2.1 implies that if $n \in N$, for any pair $x, y \in X$ there exist $r_{n}, s_{n} \in H$ and $u_{n}$, $v_{n} \in\{x, y\}$ such that

$$
\left({ }^{* *}\right) d\left(g^{n} x, g^{n} y\right) \leq P^{n}\left(d\left(r_{n} u_{n}, s_{n} v_{n}\right)\right)
$$

PROPOSITION 3.1. Let $(X ; d)$ be a $T_{2}$ semi-metric space and let $g: X \rightarrow X$. Suppose $H$ is a semigroup of self maps of $X$ such that $H \subseteq C_{B}$. If $g$ has property $P$ relative to $H$ and $F_{g}$ is nonempty and bounded, then

(i) $F_{g}$ is a singleton $\{c\}$, and (ii) $c=g(c)=h(c)$ for all $h \in H$.

PROOF. To prove (i), we first note that $h\left(F_{g}\right) \subseteq F_{B}$ for all $h \in H$. For if $h \in H$ and $a=g(a)$, then $g(h(a))=h(g(a))=h(a)$, so that $h(a) \in F_{g}$. Moreover, since $F_{g}$ is bounded, $\exists M \geq 0$ such that $d(a, c) \leq M$ for $a, c \in F_{B}$.

Now by hypothesis, $\exists c \in F_{g}$. We assert that $c$ is unique. For suppose $a \in F_{g}$. Then Note 3.1 says that we can choose $u_{n} v_{n} \in\{a, c\}$ and $r_{n}, s_{n} \in H$ such that

$$
d(a, c)=d\left(g^{n}(a), g^{n}(c)\right) \leq P^{n}\left(d\left(r_{n} u_{n}, s_{n} v_{n}\right)\right) \text {. }
$$

But since $h\left(F_{g}\right) \subseteq F_{g}$ for $h \in H$, and since $a, c \in F_{g}, r_{n} u_{n}, s_{n} v_{n} \in F_{g}$. So by the above,

$$
d\left(r_{n} u_{n}, s_{n} v_{n}\right) \leq M .
$$

Therefore, since $P$ and hence $P^{n}$ is nondecreasing, (3.1) yields

$$
\mathrm{d}(\mathrm{a}, \mathrm{c}) \leq \mathrm{P}^{\mathrm{n}}(\mathrm{M}) \text { for } \mathrm{n} \in \mathrm{N} \text {. }
$$

Since $P^{n}(M) \rightarrow 0$ as $n \rightarrow \infty$, (3.2) implies that $a=c$.

(ii) is an immediate consequence of (i), since $(h \in H) \Rightarrow h\left(F_{g}\right) \subseteq F_{g}$. Therefore, if $h \in H$, $h(c) \in\{c\} ;$ i.e, $h(c)=c$. 
COROLLARY 3.1. Let $(X ; d)$ be a bounded and complete $T_{2}$ semi-metric space. Let $\mathrm{g}$ : $X \rightarrow X$ and let $H$ be a semi-group of self maps of $X$ such that $H \subset C_{g}$ and $g(H(a)) \subset H(a)$ for $a \in X$ If $g$ has property $P$ relative to $H$, then for each $x \in X \exists$ a point $c_{x} \in X$ such that $g^{n}(x) \rightarrow c_{x}$. If $g$ is continuous at one such $c_{x}$, then $F_{g}$ is a singleton, $\{c\}$, and $c_{x}=c$ for all $x$. Moreover, $c=h(c)$ for all $h \in \mathrm{H}$.

PROOF. Since $X$ is bounded, $H(x)$ is bounded for each $x \in X$. Therefore, Theorem 2.1 implies that $g^{n}(x) \rightarrow c_{x}$ for some $c_{x} \in X$. If $g$ is continuous at one such $c_{x}$, then $g\left(c_{x}\right)=c_{x}$. But then $F_{g}$ is bounded and nonempty, so that Proposition 3.1 implies that $F_{g}=\{c\}$, a singleton, and that $c=h(c)$ for all $\mathrm{h} \in \mathrm{H}$.

Corollary 3.1 has Theorem 1.[6] by Browder and a result by Zitarosa [7] on contractive self maps of a bounded complete metric space as special cases with $\mathrm{H}=\left\{\mathrm{i}_{\mathrm{d}}\right\}$.

The proof of our next theorem, as did the proof of Corollary 2.3, requires that the union of two bounded sets be bounded. So we again need a metric. Also, observe that in Example 3.1 the set $\mathrm{H}(\mathrm{a})=$ $\{n a \cdot n \in N\}$ is unbounded for $a \neq 0$.

THEOREM 3.1. Let $g$ be a self map of a metric space (X, $d$ ) which is g-orbitally complete. Suppose that $\mathrm{H}$ is a semi-group of self maps of $\mathrm{X}$ such that $\mathrm{H} \subset \mathrm{C}_{\mathrm{g}}$ and that $\mathrm{g}$ has property $\mathrm{P}$ relative to H. If $\mathrm{g}(\mathrm{H}(\mathrm{a})) \subset \mathrm{H}(\mathrm{a})$ and $\mathrm{H}(\mathrm{a})$ is bounded for all $\mathrm{a} \in \mathrm{X}$, then $\mathrm{g}$ has a contractive point $\mathrm{c}$; i.e., $\mathrm{g}^{\mathrm{n}}(\mathbf{x}) \rightarrow$ $c$ for all $x \in X$. Moreover, $c=g(c)=h(c)$ for all $h \in H$ if $g$ is continuous at $c$

PROOF. Let $\mathrm{a} \in \mathrm{X}$. By Theorem 2.1, since $\mathrm{H}(\mathrm{a})$ is bounded, $\mathrm{g}^{\mathrm{n}}(\mathrm{a}) \rightarrow \mathrm{c}$ for some $\mathrm{c} \in \mathrm{X}$ But $g^{n}(x) \rightarrow c_{x} \in X$ for any $x \in X$ since $H(x)$ is bounded. We show $c_{x}=c$ for any $x \in X$. To this end, let $x \in X$. Then $H(x) \cup H(a)$ is bounded. Since $g$ has property $P$ relative to $H$ and $H \subset C_{g}$, Note 3.1 implies that for all $\mathrm{n} \in \mathrm{N}$ we have:

$$
d\left(g^{n}(a), g^{n}(x)\right) \leq P^{n}\left(d\left(r_{n} u_{n}, s_{n} v_{n}\right)\right)
$$

where $r_{n}, s_{n} \in H$ and $u_{n} v_{n} \in\{a, x\}$; hence, $r_{n} u_{n}, s_{n} v_{n} \in H(a) \cup H(x)$ for $n \in N$. But $H(a) \cup H(x)$ is bounded, and so $\exists M \geq 0$ such that $d\left(r_{n} u_{n}, s_{n} v_{n}\right) \leq M$ for all $n$. Thus, $P^{n}\left(d\left(r_{n} u_{n}, s_{n} v_{n}\right)\right) \leq P^{n}(M) \rightarrow$ 0 as $n \rightarrow \infty$. Hence (3.3) and the above imply:

$$
d\left(c, c_{x}\right)=\lim _{n \rightarrow \infty} d\left(g^{n}(a), g^{n}(x)\right)=0 .
$$

Thus $c=c_{x}$. If $g$ is continuous at $c$, then $c=g(c)$ But since $g^{n}(x) \rightarrow c$ for all $x, c$ is the only fixed point of $g$. Therefore, $\mathrm{c}=\mathrm{h}(\mathrm{c})$ for all $\mathrm{h} \in \mathrm{H}$ by Proposition 3.1 .

COROLLARY 3.2. Let $g$ be a self map of a metric space $(X, d)$ which is $g$-orbitally complete. Suppose that $\mathrm{O}_{\mathbf{g}}(\mathrm{x})$ is bounded for all $\mathrm{x} \in \mathrm{X}$. If $\mathrm{g}$ has property $\mathrm{P}$ relative to $\mathrm{O}_{\mathrm{g}}$, then $\exists z \in X$ such that $g^{n}(x) \rightarrow z$ for all $x \in X . \quad z$ is a unique fixed point of $g$ iff the function $F(x)=d(x, g(x))$ is lower semicontinuous at $z$.

PROOF. Since trivially, $\mathrm{O}_{\mathrm{g}} \subset \mathrm{C}_{\mathrm{g}}$ and $\mathrm{g}\left(\mathrm{O}_{\mathrm{g}}(\mathrm{x})\right) \subset \mathrm{O}_{\mathrm{g}}(\mathrm{x})$ for all $\mathrm{x} \in \mathrm{X}$, Corollary 3.2 follows immediately from Theorem 3.1 (with the observation that the last statememt is a well known consequence of " $g$ n $(x) \rightarrow z$ ")

We conclude with a theorem (rephrased) by Jachymski [5] which generalizes theorems of Rhoades and Watson [4], and which is a consequence of our Corollary 2.3.

THEOREM 2. [5] Assume that $f$ is a generalized contraction, and $(X, d)$ is f-orbitally complete. If $\lim _{n \rightarrow \infty} Q^{n}(s)=0$ for $s \in[0, \infty)$ and $\lim _{s \rightarrow \infty}(s-Q(s))=\infty$, then there exists $z \in X$ such that $f^{n} x \rightarrow z$ for any $x \in X . \quad z$ is a unique fixed point of $f$ if and only if the function $F(x)=d(x, f(x))$ is lower semicontinuous at $z$.

To see that Theorem 2. [5] does indeed follow from Corollary 2.3, first observe that (as noted before) a generalized contraction satisfies (*') of Corollary 2.3. Moreover, Lemma 3. [5] tells us that if 
$\lim _{s \rightarrow \infty}(s-Q(s))=\infty$, then the orbits $O_{f}(x)$ are bounded for all $x \in X$. Therefore, Corollary 2.3 assures us that $\exists z \in X$ such that $f^{n}(x) \rightarrow z$ for all $x \in X$. The assertion that $z$ is the unique fixed point of $f$ follows as in the proof of Corollary 3.2

4. RETROSPECT. In closing we emphasize the general nature and utility of the semi-groups $\mathrm{H}$ of self maps employed. For example, in Corollary $22 \mathrm{H}$ is any family of continuous self maps closed under composition with $\mathrm{H}(\mathrm{a})$ bounded at some one point $\mathrm{a} \in \mathrm{X}-$ no commutativity requirements are imposed. Corollary 2.3 demonstrates the utility of options provided by $\mathrm{H}$ in letting $\mathrm{H}=\mathrm{O}_{\mathrm{B}}$. And Example 1.4 indicates how, when given a map $\mathrm{g}: \mathrm{X} \rightarrow \mathrm{X}$, we can generate semigroups $\mathrm{H}$ which satisfy $\mathrm{g}(\mathrm{H}(\mathrm{a})) \subset \mathrm{H}(\mathrm{a})$.

Note also that Hausdorff semi-metric spaces (X; d) generalize metric spaces, even if the semimetric $d$ is lower semi-continuous. In fact, Cook [8] provides an example of a semi-metric space with a continuous semi-metric which is developable but not normal, and hence not a metric

A final comment. The semi-group $C_{\mathbf{g}}$ has been used to some extent in fixed point research See, e.g., $[9,10,11]$.

\section{REFERENCES}

[1] HICKS, TROY L. and RHOADES, B.E., "Fixed points for pairs of mappings in d-complete topological spaces," Internat. J. Math. and Math. Sci. 16 (1993) 259-266

[2] JUNGCK, G., "Common fixed points for noncontinuous nonself maps on nonmetric spaces," Far East. J. Math. Sci. 4(2)(1996) 199-215.

[3] JUNGCK, G., "The cohesive property," Proc. Amer. Math. Soc. 84 (1982) 138-142.

[4] RHOADES, B. E. and WATSON, B., "Generalized contractions and fixed points in metric spaces," Math. Japonica 34 (1989) 975-983.

[5] JACHYMSKI, JACEK "A generalization of the theorem by Rhoades and Watson for contractive type mappings," Math. Japonica 38 no. 6 (1993) 1095-1102.

[6] BROWDER, F. "Remarks on fixed point theorems of contractive type," Nonlinear Analysis T. M. A. $3(1979) 657-661$.

[7] ZITAROSA, A. "Una generalizzazione del teorema di Banach sulle contrazioni," Mathematıche 23 (1968) 417-424.

[8] COOK, H. "Cartesian products and continuous semi-metrics," Proceedings of Point Set Topology Conference (Editor, E. Grace), Arizona State University, Tempe, 1967

[9] JUNGCK, G. "Periodic and fixed points, and commuting mappings," Proc. Amer. Math. Soc. 76 (1979) 333-338.

[10] JUNGCK, G. "Common fixed points for commuting and compatible maps on compacta," Proc. Amer. Math. Soc. 103(1988) 977-983.

[11] ZEQING, LIU "A note on fixed point in compact metric spaces," Indian J. Math. 34 (1992) 173 176. 


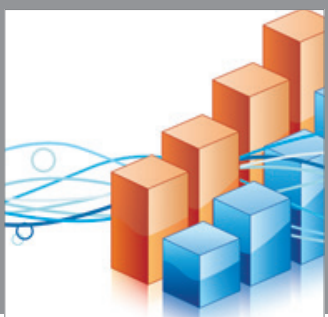

Advances in

Operations Research

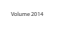

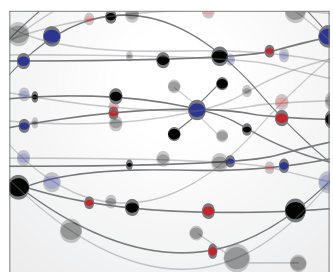

\section{The Scientific} World Journal
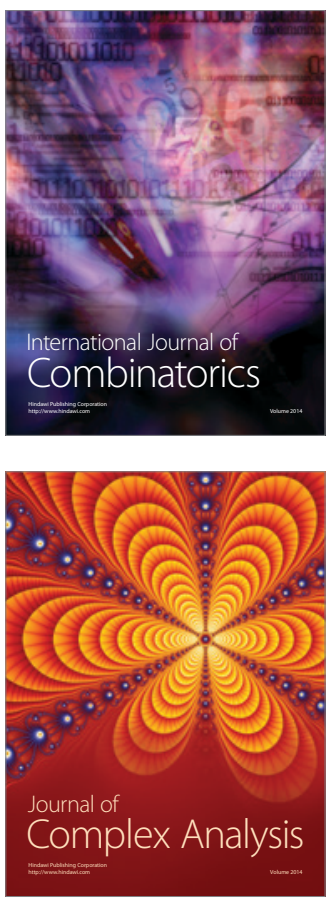

International Journal of

Mathematics and

Mathematical

Sciences
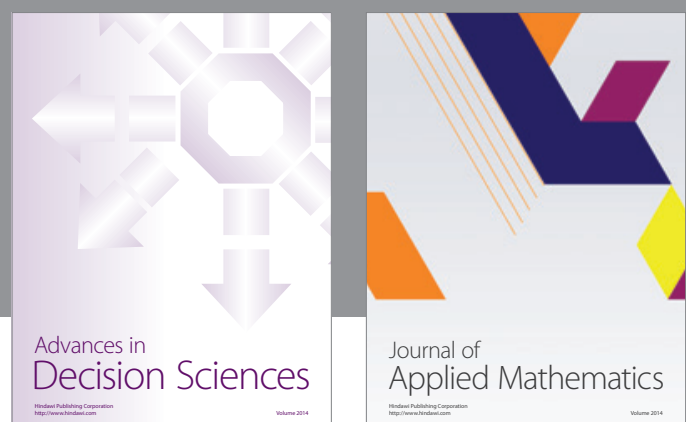

Journal of

Applied Mathematics
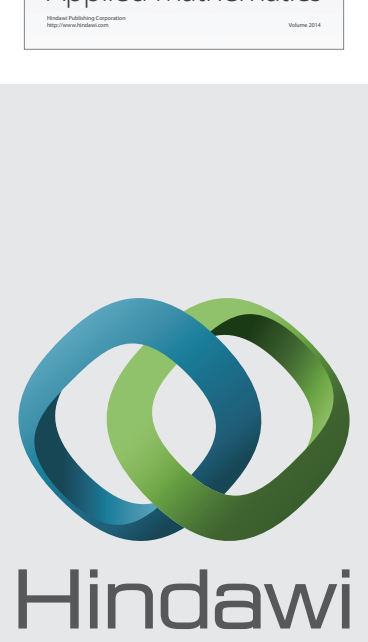

Submit your manuscripts at http://www.hindawi.com
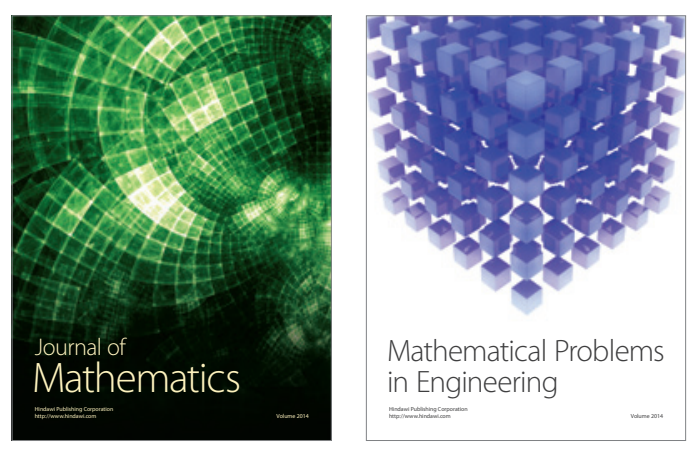

Mathematical Problems in Engineering
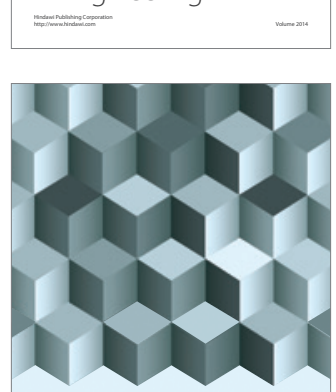

Journal of

Function Spaces
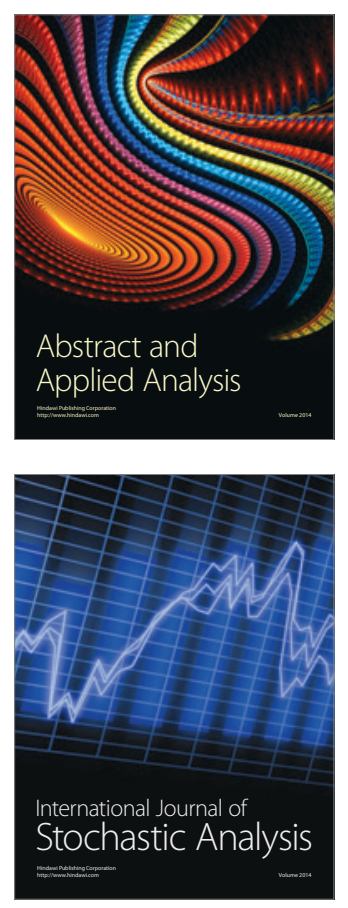

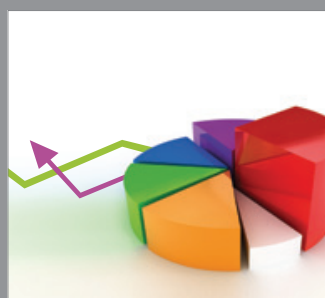

ournal of

Probability and Statistics

Promensencen
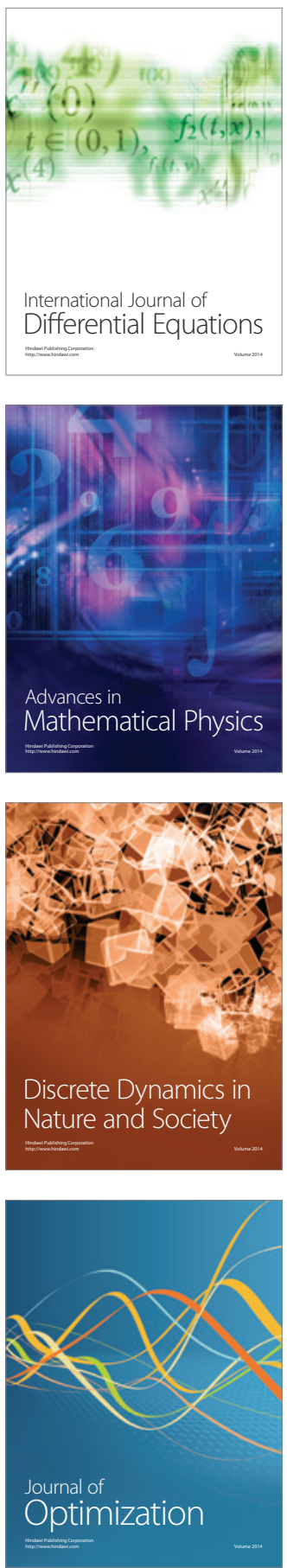